Sharif University of Technology
Scientia Iranica

\title{
Analytical study, design, and optimization of radial-flux PM limited-angle torque motors
}

\author{
R. Yazdanpanah* \\ Department of Electrical Engineering, University of Larestan, Lar, Iran.
}

Received 1 December 2019; received in revised form 25 May 2020; accepted 3 August 2020

\section{KEYWORDS}

Analytical;

Design;

Limited-angle;

Optimization;

PM;

Torque.

\begin{abstract}
This paper presents an analytical study of radial-flux slotless limited-angle torque motors. The modeling is done based on the magnetic equivalent circuit of actuators and electromagnetic equations are employed to calculate the air-gap flux as well as the produced torque. This model is then used for designing actuators both in outer-rotor and inner-rotor structures, considering the design constraints and desired characteristics. Given that the objectives in the design stage are usually in conflict with each other, an intelligent multi-objective optimization algorithm is required to design the best-fit actuators. Analytical and simulated results are presented and compared to determine the accuracy of the model and verify the design equations as well as the design approach. Given that this type of actuators is the key element in industrial control, the contributions of this paper are focused on the new analytical field solution based on magnetic equivalent circuit, design, and optimization approach to radial-flux structure and introducing a general procedure that can be extended to similar structures and actuators.
\end{abstract}

(C) 2022 Sharif University of Technology. All rights reserved.

\section{Introduction}

The Limited-Angle Torque-Motor (LATM) is an electromagnetic actuator that rotates normally in a limited angular range less than $\pm 180^{\circ}$. The conventional AC or brushless PM motors are not suitable, while LATMs have applications in servo ON-OFF valves, scan mirror systems [1], fuel control, and so on due to the inherent lack of cogging torque and high precision positioning. LATMs enjoy such advantages as higher torque per power ratio, higher reliability, lower cost, uniform torque profile, constant torque to input current ratio, and fewer mechanical parts [2-4].

Various types of LATMs have been reported in the literature. In general, two topologies of PM LATMs

\footnotetext{
*.E-mail address: ryazdanpanah@lar.ac.ir
}

include slotted and slotless armature types. Due to higher air-gap magnetic flux density, slotted LATMs have higher torque as well as cogging torque. For this type, cogging torque and partial magnetic saturation generate a non-uniform torque profile [5]. A toroidally wound stator LATM with a PM rotor does not have the cogging torque problem due to the constant reluctance path and relatively large air gap [2]. Thus, this configuration has many applications for its advantages of accurate positioning capability, low cogging torque, and high reliability [6-9].

Optimized designs employed to improve the torque capability and reduce the torque ripple are reported [5,10,11]. For slotted LATMs, stator shape optimization, e.g., asymmetrical teeth stator, and for slottless LATMs, performance optimization by the design parameters are the main methods that have been recently investigated.

In the design of electrical machines, numerical 
methods such as Finite Element Analysis (FEA) are generally used for shape optimization [12] and torque improvement [13]. Even though the analysis can be achieved using numerical methods, the analytical solutions can facilitate using an optimal design procedure $[14,15]$.

In this paper, the analytical solution based on Magnetic Equivalent Circuit (MEC) has been obtained for inner and outer rotor slotless LATMs analyses. This method is widely used in the analysis and design of electric machines $[16,17]$.

The optimization objectives of LATM include smaller size and weight as well as higher efficiency for specific output torque, whereas these objectives are usually in conflict with each other and also constraints should be taken into account in the design procedure. The optimization of LATM needs to improve several performances simultaneously. Thus, an artificial intelligence method is used to obtain the Pareto front of the objectives under design constraints.

Next, an approved solution in Pareto front is validated by FEA, where the comparison of analytical and numerical results verifies the analytical analyses and design of the structures.

Therefore, the study contributions and highlights are as follows:

- The paper focuses on a new analytical field solution based on MEC. The proposed analytical model is suitable for design of radial-flux LATMs and introduces a general design procedure considering design constraints;

- Although the proposed model is simplified, it is appropriate for the overall machine dimensions design as it reduces the model complexity and calculations. As shown by the comparison of analytical and numerical results, the proposed model enjoys acceptable accuracy and has a good correlation with exact numerical results;

- The obtained analytical model is used in an artificial intelligent algorithm to optimize the machine from the viewpoint of some performance parameters while considering constraints. Multi-objective GA optimization for two conflicting objectives is used to determine the set of designs and give full information on objective values and objective trade-offs.

\section{Machine structure}

The inner and outer rotor LATM structures and their geometrical parameters are shown in Figure 1 . The rotor holds radially magnetized PMs; neighbouring magnetic poles are magnetized in opposite directions to form a closed flux path. A toroidally wound winding and a solid core form the stator, where a range of $\mathrm{PMs}$ are covered; consequently, due to the interaction of two magnetic fields of the PM and the winding, electromagnetic toque is generated. The produced electromagnetic torque and the rotor direction of rotation are related to the magnitude and direction of the armature current. Because of the slotless structure, these actuators have no cogging torque and are thus suitable for high-precision servo control applications.

According to Figure 1, the inner rotor structure has a part of windings on the outer side of the stator that does not contribute to torque generation. Moreover, the end windings of the outer rotor actuator would be more than those of the inner rotor structure. Figure 1 shows the 2-pole design which is similar for different pole numbers.

\section{Analytical modelling}

\subsection{Magnetic flux and torque equations}

Neglecting the core reluctance, the simplified MEC

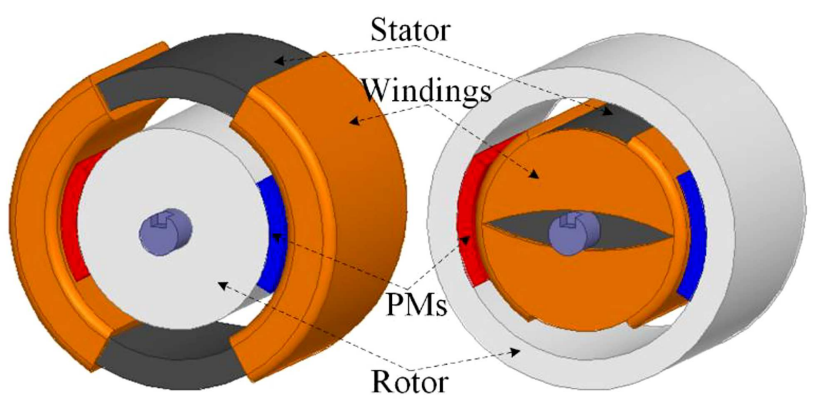

(a)

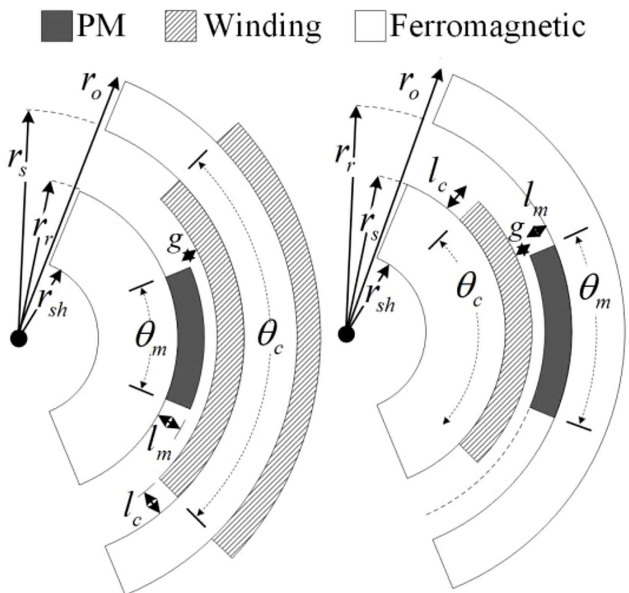

(b) (c)

Figure 1. 2-pole LATMs: (a) Inner rotor and (b) outer rotor. 


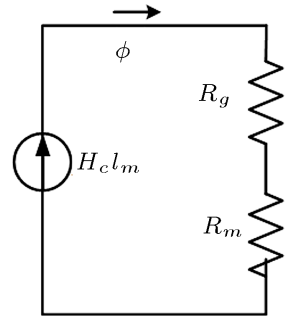

Figure 2. Simplified MEC model.

model of half north and south poles of the LATMs is shown in Figure 2.

By using this model, the air-gap magnetic flux could be calculated as follows:

$$
\phi=\frac{H_{c} l_{m}}{R_{g}+R_{m}},
$$

where:

$$
\begin{aligned}
& R_{g}=\frac{l_{g}}{\mu_{0} A_{g}}, \quad A_{g}=r_{a v g . g} \theta_{m} L, \\
& R_{m}=\frac{l_{m}}{\mu_{0} \mu_{m} A_{m}}, \quad A_{m}=r_{\text {avg.m }} \theta_{m} L,
\end{aligned}
$$

where $L$ is the axial length and:

$$
\begin{aligned}
& r_{\text {avg.g }}=\frac{r_{s}+l_{m}+r_{r}}{2} ; \text { inner rotor }, \\
& r_{\text {avg.m }}=r_{r}+\frac{l_{m}}{2} ; \text { inner rotor }, \\
& r_{\text {avg.g }}=\frac{r_{r}-l_{m}+r_{s}}{2} ; \text { outer rotor }, \\
& r_{\text {avg.m }}=r_{r}-\frac{l_{m}}{2} ; \text { outer rotor. }
\end{aligned}
$$

By using Eqs. (1)-(4), the air-gap magnetic flux results are given as follows:

$$
\begin{aligned}
& \phi=\frac{H_{c} l_{m}}{\frac{l_{g}}{\mu_{0}\left(\frac{r_{s}+l_{m}+r_{r}}{2}\right) \theta_{m} L}+\frac{l_{m}}{\mu_{0} \mu_{m}\left(r_{r}+\frac{l_{m}}{2}\right) \theta_{m} L}} ; \text { inner rotor }, \\
& \phi=\frac{H_{c} l_{m}}{\frac{l_{g}}{\mu_{0}\left(\frac{r_{r}-l_{m}+r_{s}}{2}\right) \theta_{m} L}+\frac{l_{m}}{\mu_{0} \mu_{m}\left(r_{r}-\frac{l_{m}}{2}\right) \theta_{m} L}} ; \text { outer rotor } \text {. }
\end{aligned}
$$

To calculate the produced torque, the armature current and the average air-gap radius are also required, as presented in Eq. (6). The current contribution in torque production could be calculated through the combined use of copper area and the current density. The area is obtained by the coil radius and its arch in front of the PM.

$$
\begin{aligned}
& I=\left(r_{s}-\frac{l_{c}}{2}\right) \theta_{m} l_{c} s_{f} \sigma, R_{a v g}=r_{s}-\frac{l_{c}}{2} ; \text { inner rotor }, \\
& I=\left(r_{s}+\frac{l_{c}}{2}\right) \theta_{m} l_{c} s_{f} \sigma, \quad R_{a v g}=r_{s}+\frac{l_{c}}{2} ; \text { outer rotor },
\end{aligned}
$$

where $s_{f}$ and $\sigma$ are the slot fill factor and the current density, respectively. By using Eqs. (5) and (6), the electromagnetic torque of the actuators is follows:

$$
\begin{aligned}
& T_{\text {elm }}= R_{\text {avg }} I L B=\left(r_{s}-\frac{l_{c}}{2}\right)^{2} \theta_{m} l_{c} s_{f} \sigma \times L \\
& \times \frac{1}{\left(r_{s}-\frac{l_{c}}{2}\right)} \times \frac{H_{c} l_{m}}{\frac{l_{g}}{\mu_{0}\left(\frac{r_{s}+l_{m}+r_{r}}{2}\right)}+\frac{l_{m}}{\mu_{0} \mu_{m}\left(r_{r}+\frac{l_{m}}{2}\right)}} \\
& \text { inner rotor }, \\
& T_{\text {elm }}= R_{\text {avg } I L B}=\left(r_{s}+\frac{l_{c}}{2}\right)^{2} \theta_{m} l_{c} s_{f} \sigma \times L \times \\
& \frac{1}{\left(r_{s}+\frac{l_{c}}{2}\right)} \times \frac{H_{c} l_{m}}{\frac{l_{g}}{\mu_{0}\left(\frac{r_{r}-l_{m}+r_{s}}{2}\right)}+\frac{l_{m}}{\mu_{0} \mu_{m}\left(r_{r}-\frac{l_{m}}{2}\right)}} \\
& \text { outer rotor },
\end{aligned}
$$

where $B$ is the air-gap magnetic flux density.

\subsection{Dimension constraints}

In light of the assumption of the linearity of the solutions, the average magnetic flux density in the congested area of the stator and rotor should be limited to the saturation point. The saturation point depends on the core material and the average magnetic flux density could be calculated by the magnetic flux of Eq. (5) and the cross-sectional area of the rotor and stator yokes. This criterion produces the following results:

$$
\begin{aligned}
& \frac{\phi}{2} \leq B_{\text {sat }} A_{r}=B_{\text {sat }} L\left(r_{r}-r_{s h}\right) \quad \& \\
& \frac{\phi}{2} \leq B_{\text {sat }} A_{s}=B_{\text {sat }} L\left(r_{o}-r_{s}\right) ; \text { inner rotor }, \\
& \frac{\phi}{2} \leq B_{\text {sat }} A_{s}=B_{\text {sat }} L\left(r_{s}-r_{s h}\right) \& \\
& \frac{\phi}{2} \leq B_{\text {sat }} A_{r}=B_{\text {sat }} L\left(r_{o}-r_{r}\right) ; \text { outer rotor }
\end{aligned}
$$

where $B_{\text {sat }}$ is the maximum allowable magnetic flux density.

\subsection{Power losses}

The only source of losses in LATMs is the copper loss given that the actuator is fed by direct current and the rotation is limited. The total power loss can be calculated in the following: 


$$
\begin{aligned}
& P_{\text {total }}=p \rho \sigma^{2}\left(2 l_{z} A_{z}+2 l_{\text {front }} A_{\text {front }}\right) ; \text { inner rotor }, \\
& P_{\text {total }}=\frac{p}{2} \rho \sigma^{2}\left(2 l_{z} A_{z}+2 l_{\text {front }} A_{\text {front }}\right) ; \text { outer rotor },
\end{aligned}
$$

where $p$ and $\rho$ are the number of poles and copper electrical resistivity, respectively, and:

$\left.\begin{array}{l}A_{z}=\left(r_{s}-\frac{l_{c}}{2}\right) \theta_{c} l_{c} s_{f}, \quad A_{\text {front }}=A_{z} \\ l_{\text {front }}=r_{o}-r_{s}+l_{c}, l_{z}=L+l_{c}\end{array}\right) ;$ inner rotor

and:

$$
\begin{aligned}
& A_{z}=\left(r_{s}+\frac{l_{c}}{2}\right) \theta_{c} l_{c} s_{f}, \quad A_{\text {front }}=A_{z} \\
& l_{\text {front }}=\sqrt{\left(\frac{\theta_{c}}{4} r_{s}+r_{s h}-r_{s} \cos (\alpha)\right)^{2}+\left[r_{s} \sin (\alpha)\right]^{2}}, \\
& \alpha=\frac{\pi}{2}-\frac{\theta_{c}}{4} ; \frac{\theta_{c} r_{s}}{2} \leq r_{s}-r_{s h} \\
& \text { if: } \\
& l_{\text {front }}=\sqrt{\left(\frac{r_{s}-r_{s h}}{2}+r_{s h}-r_{s} \cos (\alpha)\right)^{2}+\left[r_{s} \sin (\alpha)\right]^{2}}, \\
& \alpha=\frac{\pi}{2}-\frac{\theta_{c}}{4} ; \frac{\theta_{c} r_{s}}{2}>r_{s}-r_{s h} \\
& l_{z}=L+l_{c},
\end{aligned}
$$

for the outer rotor structure.

\section{Multi-objective design optimization}

\subsection{Optimization objectives and parameters}

As the primary design goal is to achieve the desired torque, the optimization objectives include minimizing the total volume and total loss of the actuator. Moreover, higher torque density and efficiency are the most important output parameters of the designs. Given the conflicting nature of these objectives, we try to obtain the Pareto front of the objectives.

In the present study, some parameters are held constant including core and PM parameters and some geometrical parameters, as reported in Table 1 . The pole number and the working range are set to the predefined values. So, the parameters to be decided are as follows:

$$
L, r_{o}, l_{m}, l_{c}, \theta_{c}, r_{s}\left(r_{r}\right), r_{s h} .
$$

\subsection{GA method}

GA is now extensively used for solving electromagnetic design and optimization problems since the design
Table 1. Constant design parameters.

\begin{tabular}{cccc}
\hline Parameter & Value & Parameter & Value \\
\hline$B_{\text {sat }}$ & $1.7 \mathrm{~T}$ & $g$ & $1 \mathrm{~mm}$ \\
$\rho$ & $1.7 \times 10^{-8} \Omega . \mathrm{m}$. & $P M$ & $\mathrm{NdFeB}$ \\
$s_{f}$ & 0.6 & $p$ & 2 \\
$\sigma$ & $4 \times 10^{6} \mathrm{~A} / \mathrm{m}$ & $\gamma=\theta_{c}-\theta_{m}$ & $60^{\circ}$ \\
\hline
\end{tabular}

with constraints can be considered as a constrained optimization problem [18]. The ability of GA to simultaneously search different solution space regions can find a diverse set of solutions for difficult problems with discontinuous, non-convex, and multi-modal solution spaces [19].

In this study, the multi-objective optimization problem is to minimize the fitness function defined to include two objectives that are the total volume and total loss:

$$
y=\left[\pi r_{o}^{2} L ; P_{\text {total }}\right],
$$

subject to Constraints (9) and:

$$
T_{e l m} \geq T_{d}
$$

for the optimization parameters presented in the previous sub-section.

\subsection{Optimization results}

The analytical modeling and the GA optimization are done by a MATLAB code according to the flowchart shown in Figure 3. The range of parameters is given as follows:

$$
\begin{aligned}
& 0<L<1, \quad 0<r_{o}<1, \quad 0<l_{m}<1, \quad 0<l_{c}<1, \\
& 0<r_{s}<1, \quad 0<r_{r}<1, \quad 0<r_{s h}<1 \\
& 60^{\circ}<\theta_{c}<180^{\circ} .
\end{aligned}
$$

Pareto front is a set of non-dominated solutions that are chosen as optimal if no objectives can be improved without sacrificing at least one other objective. It is conventionally shown graphically and also known as the Pareto set that can be drawn on the objective plane. In addition, it gives full information on objective values and objective trade-offs, which inform how the improvement of one objective corresponds to the deterioration of the second. The obtained Pareto fronts are shown in Figure 4.

According to Figure 4, decreasing one objective results in increasing the other, and vice versa. Also, the inner rotor structure has no valid designs in the range of $0.4-0.55$ lit, but the outer rotor structure provides lower volume and higher torque density actuators. For 


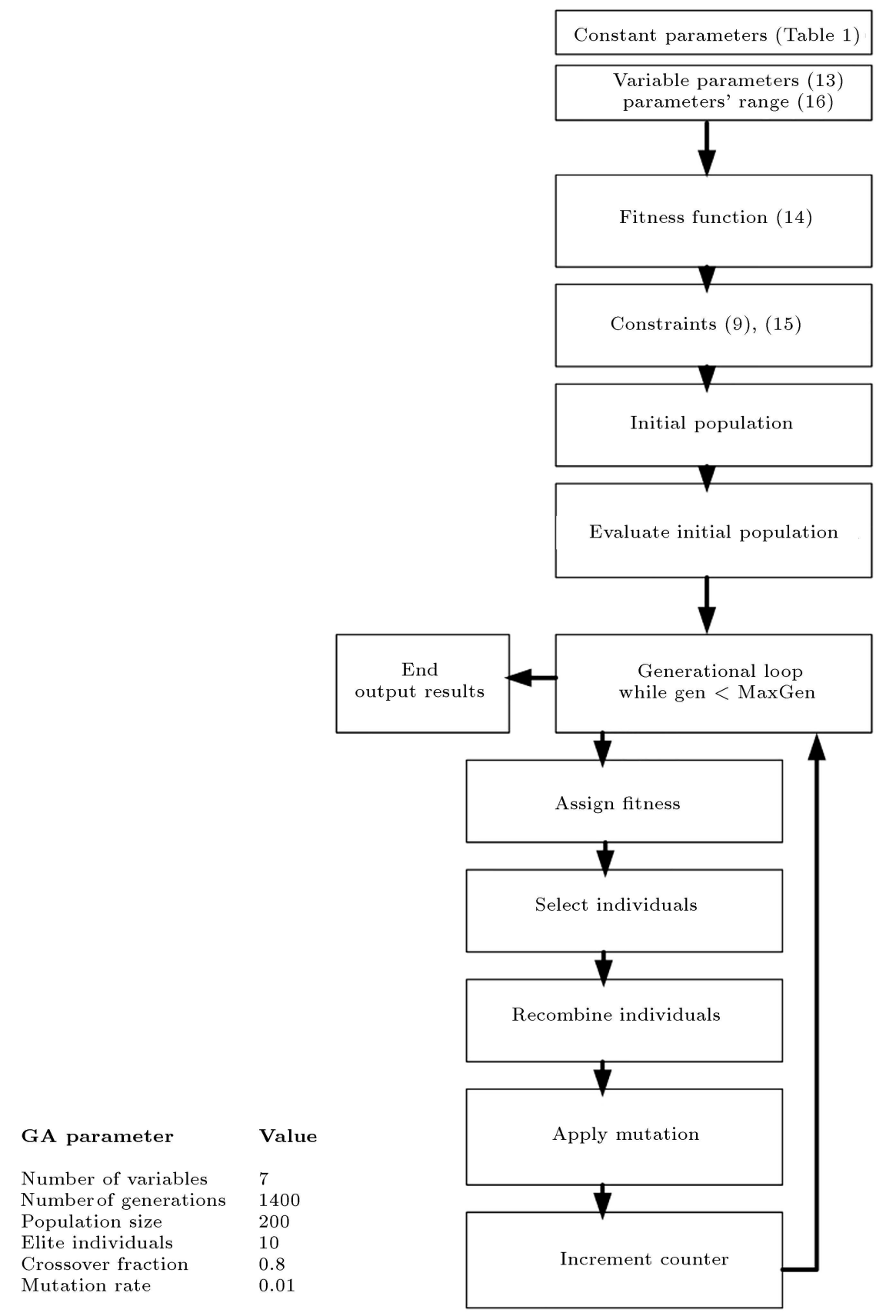

Figure 3. Multi-objective design optimization flowchart.

comparison, Table 2 presents the parameters of two actuators with the same volume and electromagnetic torque.

Figure 4 demonstrates the effectiveness of the proposed two-objective optimization based on MEC model, in which a set of machines is designed in the feasible range of the objectives. This application determines the priority of the objective of selecting one of the designed machines. The proposed approach can generally be extended to optimization of more objectives while additional constraints are considered.

\section{FEA validation of design equations}

To confirm the analytical study, inner and outer rotor structures of Table 2 are analyzed in this section by FEA. The effects of PMs and windings are simulated separately and are shown in Figure 5.

Figure 6 shows the magnitude of air-gap flux density resulting from the analytical method and FEA. The complete actuators are simulated for torque calculations, as shown in Figure 5(c) and (f). The analytical method does not calculate the magnetic flux produced by windings and thus, Figure 6(b) and (d) present only 


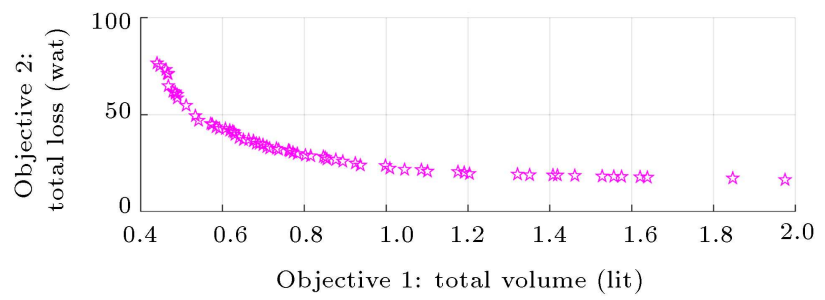

(a)

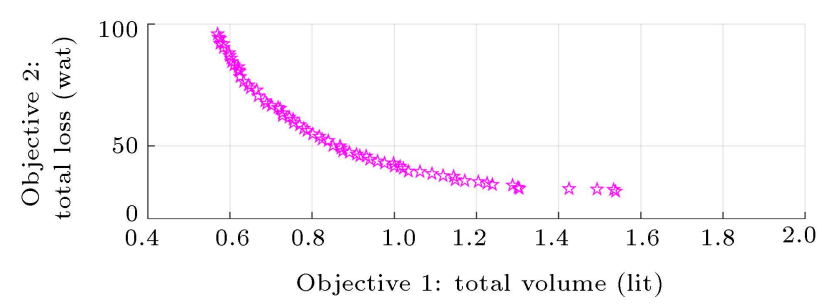

(b)

Figure 4. Pareto front of the objectives: (a) Outer rotor structure and (b) inner rotor structure.

Table 2. Comparison of two LATM structures.

\begin{tabular}{cccc}
\hline \multicolumn{2}{c}{ Inner rotor } & \multicolumn{2}{c}{ Outer rotor } \\
\hline Parameter & Value & Parameter & Value \\
\hline$r_{s h}$ & $28.5 \mathrm{~mm}$ & $r_{s h}$ & $23.2 \mathrm{~mm}$ \\
$r_{r}$ & $67.4 \mathrm{~mm}$ & $r_{r}$ & $49 \mathrm{~mm}$ \\
$l_{m}$ & $18.1 \mathrm{~mm}$ & $l_{m}$ & $18.8 \mathrm{~mm}$ \\
$l_{c}$ & $9.5 \mathrm{~mm}$ & $l_{c}$ & $12.3 \mathrm{~mm}$ \\
$\theta_{c}$ & $158^{\circ}$ & $\theta_{c}$ & $162^{\circ}$ \\
$r_{s}$ & $96 \mathrm{~mm}$ & $r_{s}$ & $81 \mathrm{~mm}$ \\
$r_{o}$ & $122 \mathrm{~mm}$ & $r_{o}$ & $101.4 \mathrm{~mm}$ \\
$L$ & $11 \mathrm{~mm}$ & $L$ & $18.3 \mathrm{~mm}$ \\
$\pi r_{o}^{2} L$ & $0.6 \mathrm{lit}$ & $\pi r_{o}^{2} L$ & $0.6 \mathrm{lit}$ \\
$P_{\text {total }}$ & $87.1 \mathrm{wat}$ & $P_{\text {total }}$ & $42.8 \mathrm{wat}$ \\
\hline
\end{tabular}

the FEA results. For this comparison, in FEA, the magnetic core is assumed to be infinitely permeable and the fluxes calculated using analytical and numerical methods have a good correlation except at the poles' edges where the fringing effect has not been considered in the analytical field solution. This correlation is also due to the negligible flux leakage as can be concluded from the flux lines of Figure 5.

The torque-angle characteristics are shown in Figure 7, where the results are coherent, especially in predicting the torque magnitude. Nevertheless, there are discrepancies concerning the torque curve where it is linear in the analytical solution and is nonlinear in FEA. This could be explained through the comparison
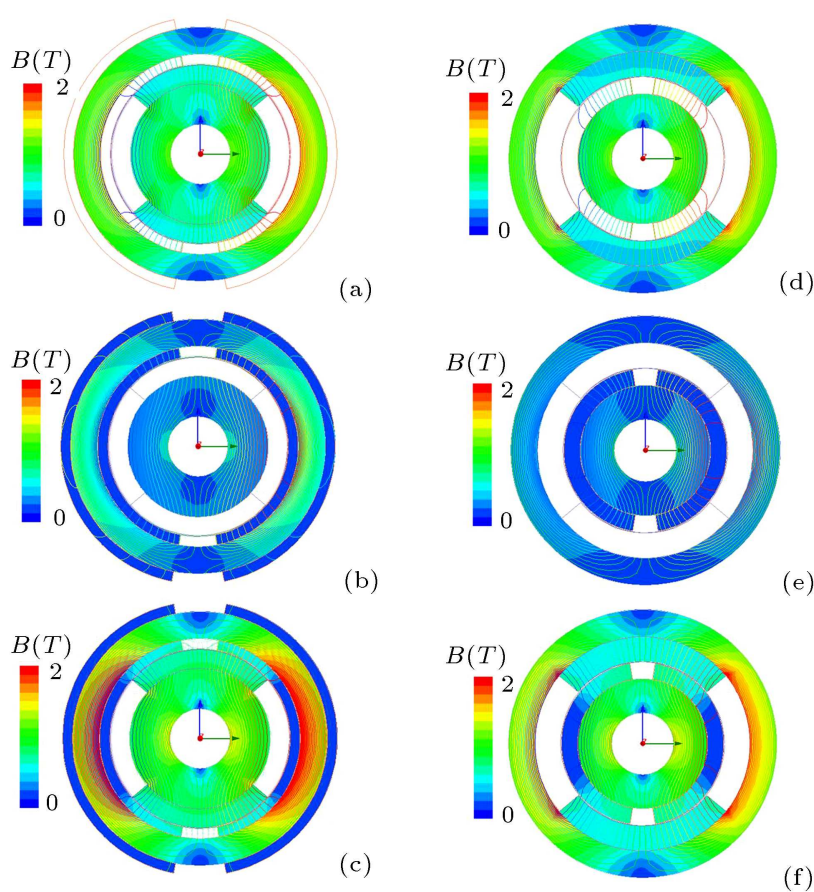

(e)

Figure 5. FEA results, inner rotor structure: (a) Excitation, (b) armature, (c) complete, outer rotor structure: (d) excitation, (e) armature, and (f) complete.

of flux density calculations at the poles' edges, as described before in Figure 6.

These figures and comparisons illustrate that analytical solutions have acceptable accuracy and this result verifies the viability of the analytical method.

\section{Conclusion}

The LATMs are electromagnetic actuators for different precise control systems with such advantages as higher torque and reliability and fewer mechanical parts. In slotless LATMs, due to uniform air-gap, the torque profile is uniform and the stator structure is simple and low-cost; hence, designing such actuators for desired characteristics remains an interesting subject for researchers.

The analytical study of the slotless and toroidally wound stator LATMs both in the case of outer rotor and inner rotor structures has been presented based on MEC analysis. This new method provides a competent way for the primary design of the LATMs including the calculation of geometrical and electromagnetic parameters considering design constraints for the desired characteristics. The simplified MEC model is appropriate for the overall optimization of machine dimensions as it reduces the model complexity and calculations. Nevertheless, for the machine under study which is slotless and the PMs are surface mounted, the proposed MEC model results are in good correlation with exact numerical ones in terms of air-gap magnetic 


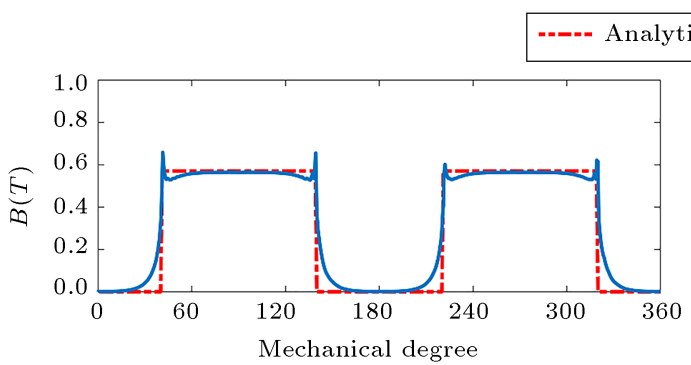

(a)

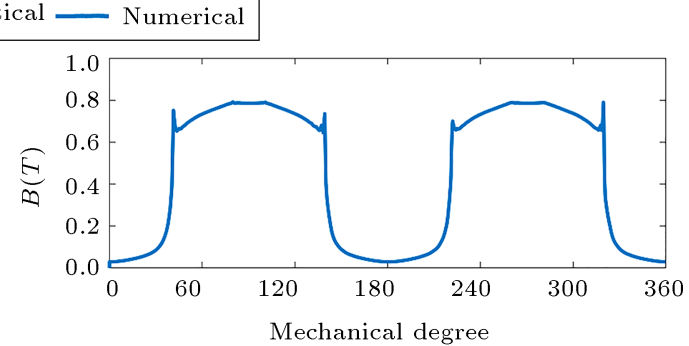

(b)

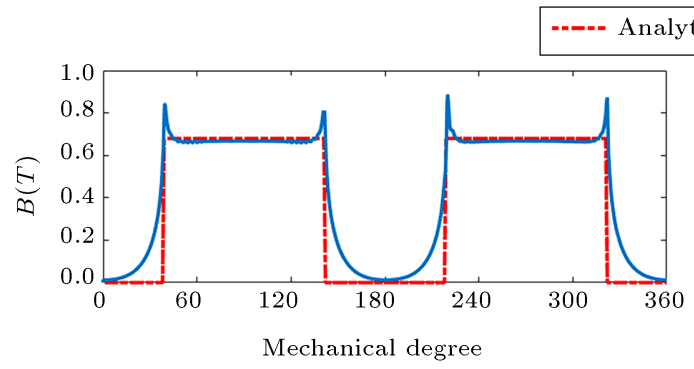

(c)

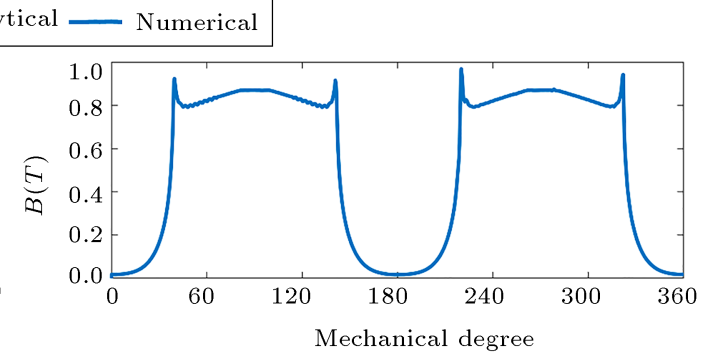

(d)

Figure 6. Comparison of the analytical and numerical results, inner rotor structure air-gap magnetic flux density by: (a) PMs, (b) PMs and windings, outer rotor structure air-gap magnetic flux density, (c) PMs, and (d) PMs and windings.

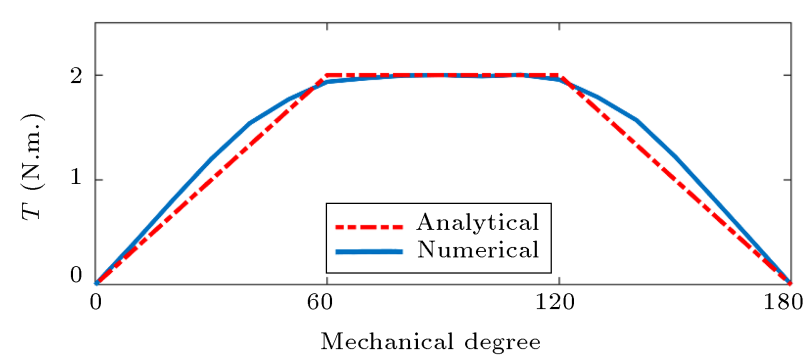

(a)

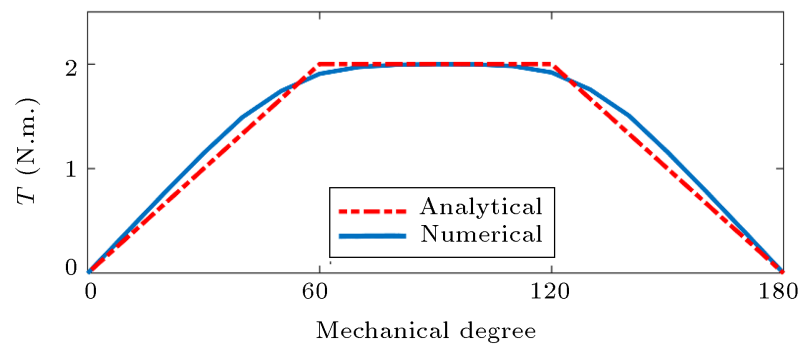

(b)

Figure 7. Comparison of the analytical and numerical results: (a) Inner rotor structure and (b) outer rotor structure.

flux density and the produced electromagnetic torque. This finding may not hold for other structures for which more detailed MEC may be required.

This analysis method was used to optimize the actuators for two conflicting objectives and to find the Pareto front of the objectives. Also, FEA as a tool for realizing the electromagnetic structures was employed to validate the design equations and approach. The comparison of the results verifies the ability of the approach to the optimal design of radial-flux slotless LATMs and its application to similar structures.

\section{Nomenclature}

$\phi \quad$ Air-gap magnetic flux

$B \quad$ Air-gap magnetic flux density

$B_{\text {sat }} \quad$ Maximum allowable magnetic flux density

I Armature current

$T_{\text {elm }} \quad$ Electromagnetic torque

$T_{d} \quad$ Desired torque

$P_{\text {total }} \quad$ Total power loss

$H_{c} \quad$ PM magnetic coercivity

$R_{g}, R_{m} \quad$ Air-gap and PM reluctance

$A_{r}, A_{s} \quad$ Rotor and stator yoke cross-section area

$r_{s}, r_{r} \quad$ Stator and rotor radius

$L \quad$ Axial length

$R_{\text {avg }} \quad$ Average air-gap radius

$p \quad$ Number of poles

$\rho \quad$ Copper electrical resistivity

$s_{f} \quad$ Slot fill factor

$\sigma \quad$ Current density

$r_{o} \quad$ Outer radius

$l_{m} \quad$ PM thickness

$l_{c} \quad$ Coil thickness

$\theta_{c} \quad$ Coil angle 
$\theta_{m}$

PM angle

\section{References}

1. Chen, S., Kamaldin, N., Teo, T., et al. "Toward comprehensive modeling and large-angle tracking control of a limited-angle torque actuator with cylindrical Halbach", IEEE/ASME Trans. Mechatronics, 21(1), pp. 431-442 (2016).

2. Hekmati, P., Yazdanpanah, R., Mirsalim, M., et al. "Radial-flux permanent-magnet limited-angle torque motors", IEEE Trans. Ind. Electron., 64(3), pp. 18841892 (2017).

3. Guodong, Y., Yongxiang, X., Jibin, Z., et al. "Analysis and experimental validation of dynamic performance for slotted limited-angle torque motor", IEEE Trans. Magn., 53(11) (2017).

4. Zhang, Y., Smith, I.R., and Kettleborough, J.G. "Accurate tracking control of a limited angle torque motor", Electric Machines \& Power Systems, 27(11), pp. 1191-1199 (1999).

5. Yu, G., Yongxiang, X., Zou, J., et al. "Development of a radial-flux slotted limited-angle torque motor with asymmetrical teeth for torque performance improvement", IEEE Trans. Magn., 55(7) (2019).

6. Hekmati, P. and Mirsalim, M. "Design and analysis of a novel axial-flux slotless limited-angle torque motor with trapezoidal cross section for the stator", IEEE Trans. Energy Convers., 28(4) (2013).

7. Tsai, C.C., Lin, S.C., Huang, H.C., et al. "Design and control of a brushless DC limited-angle torque motor with its application to fuel control of small-scale gas turbine engines", Mechatronics, 19(1), pp. 29-41 (2009).

8. Nasiri-Zarandi, R., Mirsalim, M., and Cavagnino, A. "Analysis, optimization, and prototyping of a brushless DC limited-angle torque-motor with segmented rotor pole tip structure", IEEE Trans. Ind. Electron., 62(8), pp. 4985-4993 (2015).

9. Roohnavazfar, M., Houshmand, M, Zarandi, R.N., et al. "Optimization of design parameters of a limited angle torque motor using analytical hierarchy process and axiomatic design theory", Production \& Manufacturing Research, 2(1), pp. 400-414 (2014).

10. Xue, Z., Li, H., Zhou, Y., et al. "Analytical prediction and optimization of cogging torque in surface-mounted permanent magnet machines with modified particle swarm optimization", IEEE Trans. Ind. Electron., 64(12), pp. 9795-9805 (2017).

11. Arehpanahi, M. and Kashefi, H. "Cogging torque reduction of Interior permanent magnet synchronous motor (IPMSM)", Scientia Iranica, 25(3), pp. 14711477 (2018).

12. Yamazaki, K. and Ishigami, H. "Rotor-shape optimization of interior permanent-magnet motors to reduce harmonic iron losses", IEEE Trans. Ind. Electron., 57(1), pp. 61-69 (2010).

13. Ibtissam, B., Mourad, M., Medoued, A., et al. "Multi-objective optimization design and performance evaluation of slotted Halbach PMSM using Monte Carlo method", Scientia Iranica, 25(3), pp. 1533-1544 (2018).

14. Yazdanpanah, R. and Mirsalim, M. "Hybrid electromagnetic brakes: design and performance evaluation", IEEE Trans. Energy Convers., 30(1), pp. 60-69 (2015).

15. Chen Xing, Deng Zhaoxue, Hu Jibin, et al. "An analytical model of unbalanced magnetic pull for PMSM used in electric vehicle: numerical and experimental validation", International Journal of Applied Electromagnetics and Mechanics, 54(4), pp. 583-596 (2017).

16. Mohammadi Ajamloo, A., Abbaszadeh, K., and NasiriZarandi, R. "A novel transverse flux permanent magnet generator for small-scale direct drive wind turbine application: design and analysis", Scientia Iranica, 28(6), pp. 3363-3378 (2021).

17. Liu, Y., Zhang, M., Zhu, Y., et al. "Optimization of voice coil motor to enhance dynamic response based on an improved magnetic equivalent circuit model", IEEE Trans. Magn., 47(9), pp. 2247-2251 (2011).

18. Wurtz, F., Richomme, M., Bigeon, J., et al. "A few results for using genetic algorithms in the design of electrical machines", IEEE Trans. Magn., 33(2), pp. 1892-1895 (1997).

19. Konaka, A., Coitb, D.W., and Smithc, A.E. "Multiobjective optimization using genetic algorithms: a tutorial", Reliability Engineering and System Safety, 91(9), pp. 992-1007 (2006).

\section{Biography}

Reza Yazdanpanah (M'14) received his MSc degree from the Isfahan University of Technology and his $\mathrm{PhD}$ degree in Electrical Power Engineering from the Amirkabir University of Technology (Tehran Polytech.) in 2006 and 2014, respectively. He is currently a faculty member at the Department of Electrical Engineering, University of Larestan. His research interests include electromagnetic systems analysis, design and analysis of electrical machines \& drives, power electronics, and applied nonlinear control. 